\title{
Analysis of results from the Joint External Evaluation: examining its strength and assessing for trends among participating countries
}

\author{
Vin Gupta ${ }^{1,2,3}$, \\ John D Kraemer ${ }^{4}$, \\ Rebecca Katz ${ }^{5}$, \\ Ashish K Jha ${ }^{6}$, \\ Vanessa B Kerry ${ }^{7,8,9}$, \\ Jussi Sane ${ }^{10}$, \\ Jukka Ollgren ${ }^{10}$, \\ Mika O Salminen ${ }^{10}$ \\ ${ }^{1}$ Division of Pulmonary and Critical Care \\ Medicine, Brigham \& Women's Hospital, \\ Boston, Massachusetts, USA \\ 2 Harvard Global Health Institute, Harvard \\ University, Cambridge, Massachusetts, USA \\ ${ }^{3}$ Institute for Health Metrics and Evaluation, \\ Seattle, Washington, USA \\ ${ }^{4}$ Georgetown University, Department of Health \\ Systems Administration, Washington, D.C., USA \\ ${ }^{5}$ Georgetown University, Center for Global \\ Health Science and Security, Washington, D.C., \\ USA \\ ${ }^{6}$ Department of Health Policy, Harvard T.H. \\ Chan School of Public Health, Boston, \\ Massachusetts, USA \\ ${ }^{7}$ Department of Global Health and Social \\ Medicine, Harvard Medical School, Boston, \\ Massachusetts, USA \\ ${ }^{8}$ MGH Global Health, Massachusetts General \\ Hospital, Boston, Massachusetts, USA \\ ${ }^{9}$ Seed Global Health, Boston, Massachusetts, \\ USA \\ ${ }^{10}$ Department for Health Security, National \\ Institute for Health and Welfare (THL), Helsinki, \\ Finland
}

\section{Correspondence to:}

Vin Gupta, MD, MPA

Assistant Professor of Global Health

Division of Health Metrics Sciences

23015 th Avenue

Suite 600

Seattle, WA 98121

United States of America

vgupta7@bwh.harvard.edu
Background The Joint External Evaluation (JEE) is part of the World Health Organization's (WHO) new process to help countries assess their ability to prevent, detect and respond to public health threats such as infectious disease outbreaks, as specified by the International Health Regulations (IHR). How countries are faring on these evaluations is not well known and neither is there any previous assessment of the performance characteristics of the JEE process itself.

Methods We obtained JEE data for 48 indicators collectively across 19 technical areas of preparedness for 55 countries. The indicators are scored on a 1 to 5 scale with 4 indicating demonstrated capacity. We created a standardized JEE index score representing cumulative performance across indicators using principal components analysis. We examined the state of performance across all indicators and then examined the relationship between this index score and select demographic and health variables to better understand potential drivers of performance.

Results Among our study cohort, the median performance on 43 of the 48 (89.6\%) indicators was less than 4 , suggesting that countries were failing to meet demonstrated capacity on these measures. The two weakest indicators were related to antimicrobial resistance (median score $=1.0$, interquartile range $=1.0-2.0$ ) and biosecurity response (median score $=2.0$, interquartile range $=2.0-3.0$ ). JEE index scores correlated with various metrics of health outcomes (life expectancy, under-five year mortality rate, disability-adjusted life years lost to communicable diseases) and with standard measures of social and economic development that enable public health system performance in the total sample, but in stratified analyses, these relationships were much weaker in the AFRO region.

Conclusions We find large variations in JEE scores among countries and WHO regions with many nations still unprepared for the next disease outbreak with pandemic potential The strong correlations between JEE performance and metrics of both health outcomes and health systems' performance suggests that the JEE is likely accurately measuring the strength of IHR-specific, public health capabilities.

Building global capacity to prevent, detect and respond to epidemics with pandemic potential has proven difficult $[1,2]$. The revised International Health Regulations (IHR), adopted in 2005, obligated all Member States of the World Health Assembly to build capacities to detect, assess, notify, and respond to potential public health emergencies in a timely manner $[3,4]$. Weak accountability mechanisms to ensure compliance and lack of 
dedicated resources have complicated these efforts and are widely seen as part of the explanation for the poor local and global response to the Ebola outbreak in western Africa [5].

Preparing for population level health threats, such as infectious disease outbreaks, requires sustained investments in certain public health functions, including preventive interventions (eg, immunization programs, food and waterborne disease control measures), physical infrastructure (eg, laboratory and surveillance capabilities), trained personnel (eg, health professionals, epidemiologists) [6-8], and ongoing commitments to resources for supplies, maintenance, and training [9]. Given competing resources, relatively few countries have made consistent investments in these areas and a majority have been unable to meet their obligations under the IHR. Major donors of health aid have often focused their attention elsewhere as well $[10,11]$. While investment in these population level preventive functions is generally regarded as highly cost-effective, investments in curative health services are frequently prioritized due to high demand and greater visibility [12].

In 2010, the World Health Organization (WHO) identified 13 core capacities derived from Annex 1 of the IHR for the purpose of monitoring capacity building efforts and compliance. These core capacities were used by countries to complete self-assessments and self-reporting to $\mathrm{WHO}$, but these assessments were criticized for lack of transparency and not representing the true measure of capacity for health security within countries $[13,14]$. Although variation exists, analyses of the reported self-assessed country data suggests probable overestimation of real capacity in many areas of the world (Tsai and Katz, submitted manuscript). Following the 2014-2016 Ebola outbreak in West Africa and the spread of Middle Eastern Respiratory Syndrome (MERS) to the Republic of Korea in 2015, and in response to recommendations provided by the IHR review committee [13], the WHO developed (through a regional consultation process) a new approach to monitoring and evaluation of the IHR [15-17]. As part of this new approach, the WHO adopted the Joint External Evaluation (JEE) process and tool [18] in January 2016 for broad voluntary use in assessing the ability of a nation to prevent, detect and respond to a disease of pandemic potential [19-21].

The JEE tool includes 19 technical areas with 48 indicators. A globally standardized process, the first step of a JEE is a self-assessment by an internal country team; these results are then compared and validated against a separate external evaluation conducted by a team of independent substance experts. Ultimately, the JEE results are supposed to contribute to the development of costed and financed multisectoral national action plans designed to address any prioritized gaps in a country's capacity to prevent, detect and respond to public health threats [22]. Now two years into its implementation, there has been scant examination of country-level results from the JEE.

In this study, we used the most recent data available on JEE assessments to answer two questions: First, how are assessed countries performing across all indicators and specific technical areas within the JEE and what are the regional variations in performance? Second, as a new process, do JEE scores correlate in expected ways with population-level health outcomes (results of public health capacities) and inputs (determinants of public health capacities). In other words, does the JEE accurately measure the core public health capacities it intends to assess?

\section{METHODS}

\section{Data sources}

Joint External Evaluation (JEE) data was extracted from April 1, 2017 to March 4, 2018; 55 countries' data was available (Appendix S1 in Online Supplementary Document). Data was gathered from the WHO Strategic Partnership Portal (https://extranet.who.int/spp/ihr-monitoring-evaluation) [23], which provides a full overview and continuous updates of JEEs, published mission reports and other information on WHO supported activities to strengthen IHR implementation. The process for assessment via the JEE has been fully described previously [24].

Online databases from the Institute for Health Metrics and Evaluation (IHME) and the World Bank provided relevant health, demographic, and economic descriptive statistics for each country in our study sample. Global burden of disease (GBD) data from 2015, as measured in disability-adjusted life years (DALYs) per 100000 persons, was available from the IHME (http://www.healthdata.org/results/data-visualizations). The most recent figures on population size, gross domestic product (GDP) per capita, public health expenditures as a percentage of GDP, life expectancy, under 5-year mortality, and density of skilled health professionals were available from the World Bank (http://databank.worldbank.org/data/). 


\section{Definitions}

The JEE tool is divided into 19 technical areas (representing public health functions relevant to health security) represented by 48 separate indicators (1 to 5 per technical area) (Appendix S2 in Online Supplementary Document). Scores for each of the 19 technical areas represent the mean of the scores for the indicators of that area. Each indicator in the JEE is scored on a five-point ordinal scale. A score of 1 reflects no pertinent capacity, 2 reflects limited capacity, 3 notes developed capacity, 4 connotes demonstrated capacity, and a score of 5 reflects sustainable capacity (Appendix S3 in Online Supplementary Document). For the purposes of our analysis, we identified a score of 4 as representing a minimum level of desired target performance, as we believe this indicates functionality and, at least, medium-term sustainability in a specific indicator.

To assess concordance with JEE performance, we chose three indicators that measure national health outcomes and three which measure inputs that enable public health system performance. The measures chosen to examine correlations with selected health outcomes were life expectancy at birth (years), under 5-year mortality rate (per 1000 live births) and disability-adjusted life years lost (DALYs) due to communicable disease (per 100000 persons). These were selected as general population health, childhood health and communicable disease burden indicators. The three indicators that represent enabling factors were GDP per capita (in US\$), public health expenditures as a percentage of GDP, and density of skilled health professionals (per 10000 persons). Other indicators were available, but for the purposes of conciseness we decided to limit our study to one indicator each representing various aspects of adult, childhood and communicable disease-specific population health statuses, general economic development levels, investment in public health, and the availability of health professionals. Indicators were subjectively chosen based on frequent use for various international benchmarking analyses $[25,26]$.

\section{Analyses}

We produced basic descriptive statistics (medians and interquartile ranges) for various baseline demographic variables both for the overall data set and stratified by WHO region, merging the regional offices for Europe (EURO) and the Americas (PAHO) because few JEEs have been conducted in those regions. We assessed differences in these variables across regions using the Kruskall-Wallis test. Similar descriptive statistics were reported for each of the 48 indicators comprising the 19 technical areas of the JEE. The distribution of medians for these 48 indicators are presented in histogram format, with results stratified by WHO region.

In order to assess how representative our 55-country sample was of all 196 WHO states parties, we assessed for differences among the above baseline variables between those countries that did and did not complete the JEE. These variables were: population, GDP per capita, life expectancy, public health expenditures as a share of GDP (\%), under-5-year mortality rate, DALYs due to communicable diseases, and density of skilled health professionals. Differences in the distributions of the variables were examined using the Mann-Whitney U-test and results were stratified by WHO region.

Next, we examined whether JEE performance correlated with population health outcomes and enabling factors as expected. To do so, we first created a JEE index score by applying principal components analysis to a polychoric correlation matrix, which correctly handles ordinal variables, of the 19 individual technical area scores [27]. Using a scree plot, we determined that there was only one principal component (which explained $71 \%$ of the variance and which was the only component with an eigenvalue greater than 1 ). This component was retained; its score was calculated and then standardized for ease of interpretation. We then assessed correlations between the JEE index score and variables representing each enabling factor and health outcome using Spearman's rank-order correlation and calculated bias-corrected and accelerated percentile confidence intervals using 10000 bootstrap replicates [28]. We analyzed region-specific correlations for AFRO and EMRO, the regions with the largest samples, to assess whether there were contextual differences in associations.

We conducted descriptive analyses using Microsoft Excel; all other analyses used Stata version 15.1 (Stata Corp, College Station, TX, USA). Statistical code to replicate the analysis is included as Appendix S1 in Online Supplementary Document.

\section{Patient involvement}

This study did not involve patients in its design, nor were the development of outcome measures informed by patients' experiences or preferences. Our institutions did not require human subjects review because the study involved no interaction with human subjects or use of their data. 


\section{RESULTS}

Fifty-five of the 196 IHR states parties have completed JEE assessments that were publically available for review as of March 4, 2018. Twenty-three (41.8\%) of the analyzed countries are from the WHO Africa region, 13 from the Eastern Mediterranean (23.6\%), eight from Europe (14.5\%), five from Southeast Asia (9.1\%), five (9.1\%) from the Western Pacific, and one from the Americas (1.8\%). Together, the 55-nation sample represents $25.8 \%$ ( $n=1.88$ billion) of the world population, and $69.1 \%(n=38)$ are classified as low or lower-middle income by the World Bank (by comparison, $42.8 \%$ or $\mathrm{n}=83$ of all WHO-member countries are classified as lower or lower-middle income). The distribution of demographic, economic and health variables among these regions are reported in Table 1. Among our study sample, significant differences were observed across regions, with countries in AFRO reporting the poorest levels of performance across these various factors. Within each region, the countries that have undergone the JEE were observed, with two exceptions, to not have significant differences across these baseline variables when compared to those countries who have yet to undergo the assessment (Table 2).

Forty-three of the 48 (89.6\%) total indicators included in the JEE assessment had a median score of $<4$ across the 50 countries analyzed. Analyzed by region, 46 (95.8\%) indicators had a median score $<4$ in AFRO, 7 (14.6\%) in PAHO+EURO, 37 (77.1\%) in EMRO, 40 (83.3\%) in SEARO, and 39 (81.3\%) in WPRO (Figure 1).

The following indicators had the lowest median scores across the sample: antimicrobial stewardship activities, surveillance of infections caused by antimicrobial resistance (AMR), and the presence of biosecurity systems. The highest median indicator values were the presence of national vaccine access and delivery protocols, laboratory testing capabilities for priority diseases, and syndromic surveillance systems.

Statistically significant correlations were observed in the full sample between the JEE index score and life expectancy (Spearman's $\rho=0.84,95 \% \mathrm{CI}=0.76$ to 0.90), DALYs lost to communicable diseases $(\rho=-0.83$, $95 \% \mathrm{CI}=-0.90$ to -0.75$)$, and under -5 mortality rate per 1000 live births $(\rho=-0.87,95 \%=\mathrm{CI}-0.92$ to -0.78). In AFRO, the only statistically significant relationship with health outcomes was with the under-5 mortality rate $(\rho=-0.59,95 \% \mathrm{CI}=-0.81$ to -0.26$)$ (Table 3 and Figure 2$)$. In EMRO, JEE performance
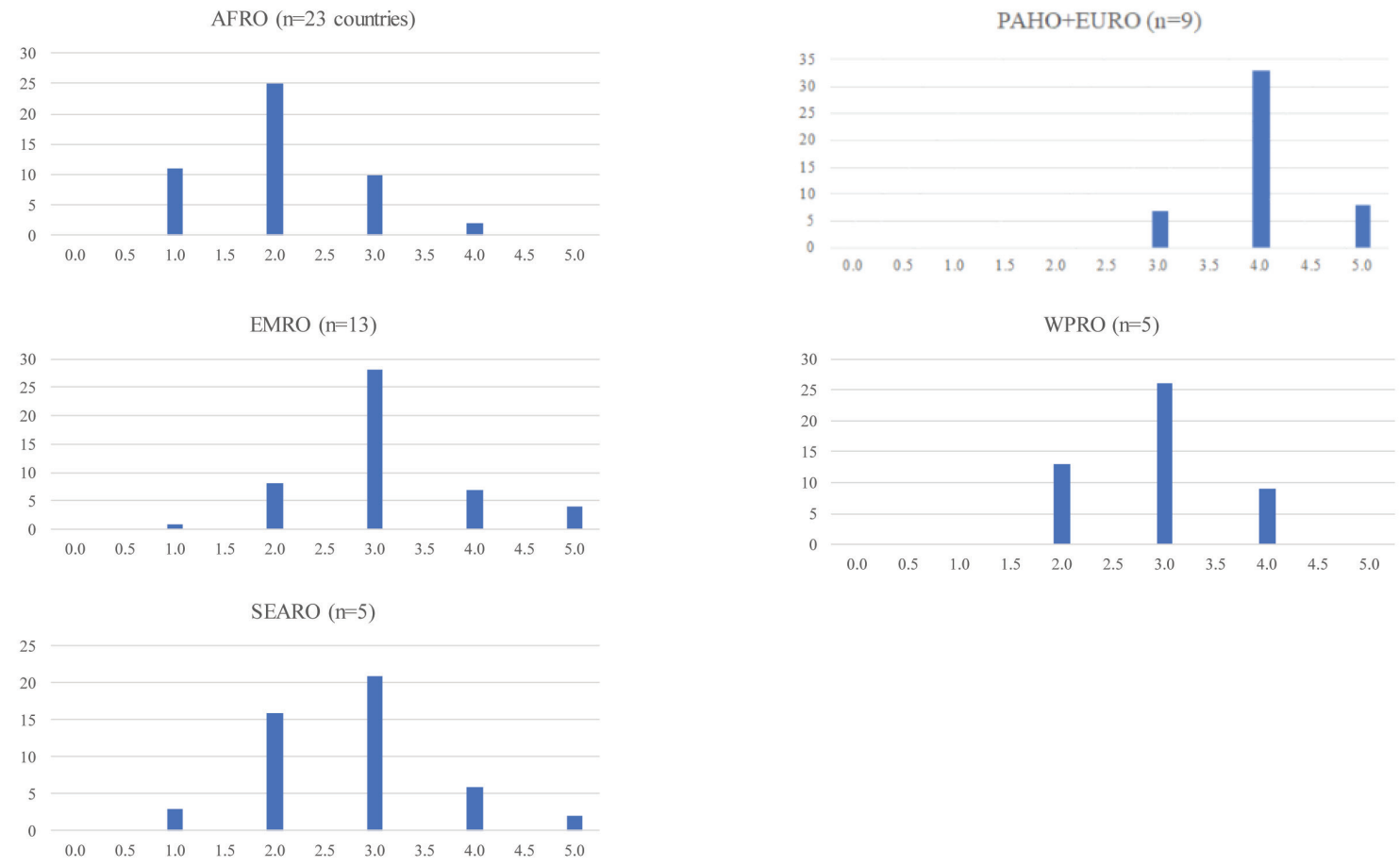

Figure 1. Distribution of median performance on 48 JEE Indicators across the 6 WHO regions. 


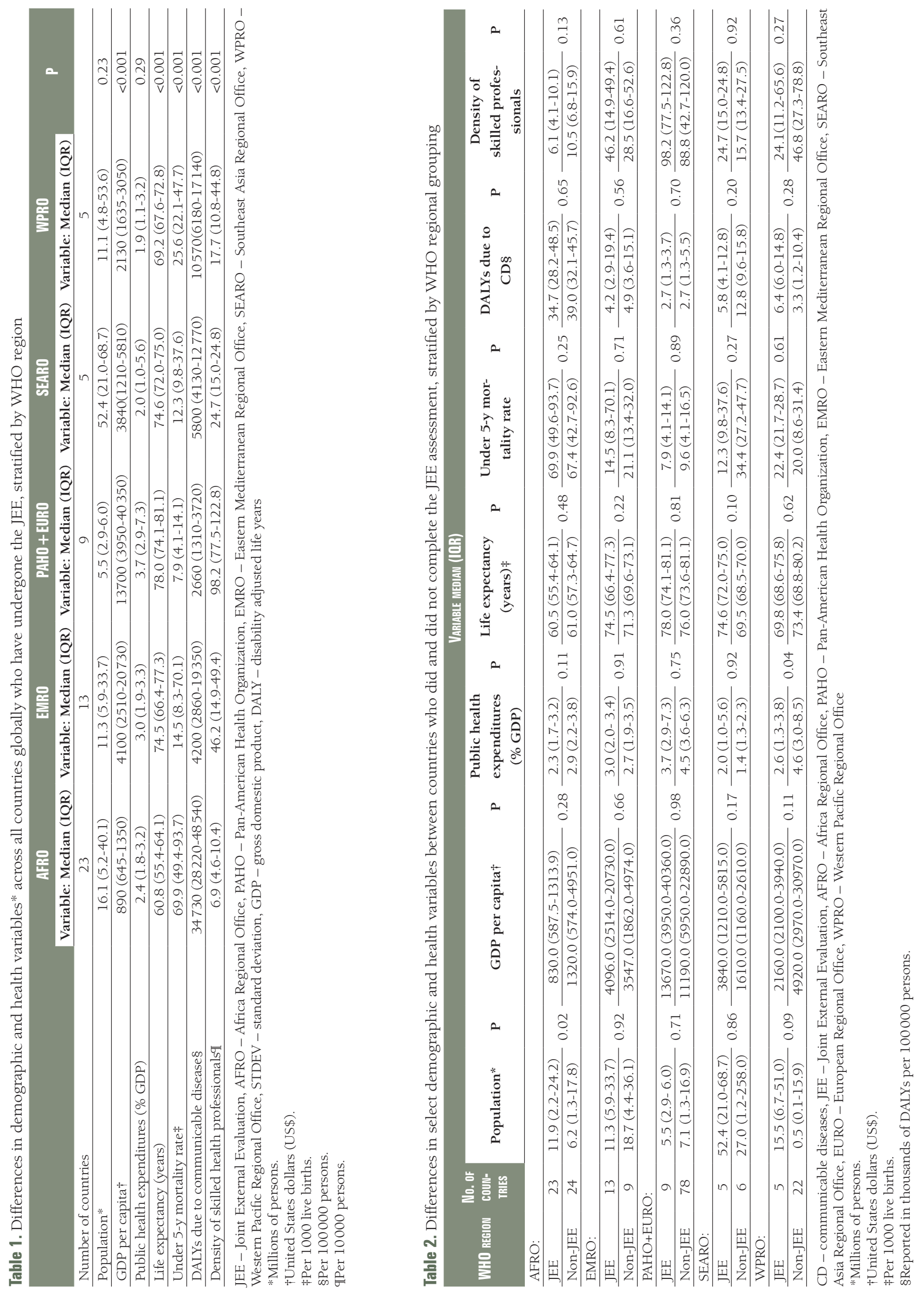


Table 3. Spearman rank-order correlation of various health outcomes and enabling factors with standardized score of JEE performance in the overall sample and stratified to the AFRO and EMRO regions

\begin{tabular}{|c|c|c|c|}
\hline & \multicolumn{3}{|c|}{ Spearman's Rho (95\% confidence InterVal)* } \\
\hline Health or economic outcome & Overall ( $\mathrm{n}=55$ countries $)$ & $\operatorname{AFRO}(n=23)$ & $\operatorname{EMRO}(n=13)$ \\
\hline Life expectancy at birth (years) & $0.84(0.76$ to 0.90$)$ & $0.34(-0.05$ to 0.66$)$ & $0.66(-0.15$ to 0.97$)$ \\
\hline Lost DALYs attributable to communicable diseases & $-0.83(-0.90$ to -0.75$)$ & $-0.17(-0.52$ to 0.21$)$ & $-0.65(-0.92$ to -0.01$)$ \\
\hline Under-5 mortality rate (per 1000 live births) & $-0.87(-0.92$ to -0.78$)$ & $-0.59(-0.81$ to -0.26$)$ & $-0.80(-0.97$ to -0.25$)$ \\
\hline \multicolumn{4}{|l|}{ Enabling factors: } \\
\hline Gross domestic product per capita (US\$) & $0.81(0.68$ to 0.89$)$ & $0.17(-0.25$ to 0.55$)$ & 0.85 (0.61 to 0.99$)$ \\
\hline Public health (\% of GDP) & $0.36(0.10$ to 0.58$)$ & $0.10(-0.33$ to 0.49$)$ & $0.26(-0.35$ to 0.73$)$ \\
\hline Density of skilled health professionals & $0.81(0.71$ to 0.89$)$ & $0.07(-0.46$ to 0.54$)$ & $0.51(-0.15$ to 0.90$)$ \\
\hline
\end{tabular}

DALY - Disability-Adjusted Life Years, AFRO - Africa Regional Office, EMRO - Eastern Mediterranean Regional Office, GDP - Gross Domestic Product *Bias-corrected and accelerated percentile 95\% confidence intervals based on 10000 bootstrap replications.

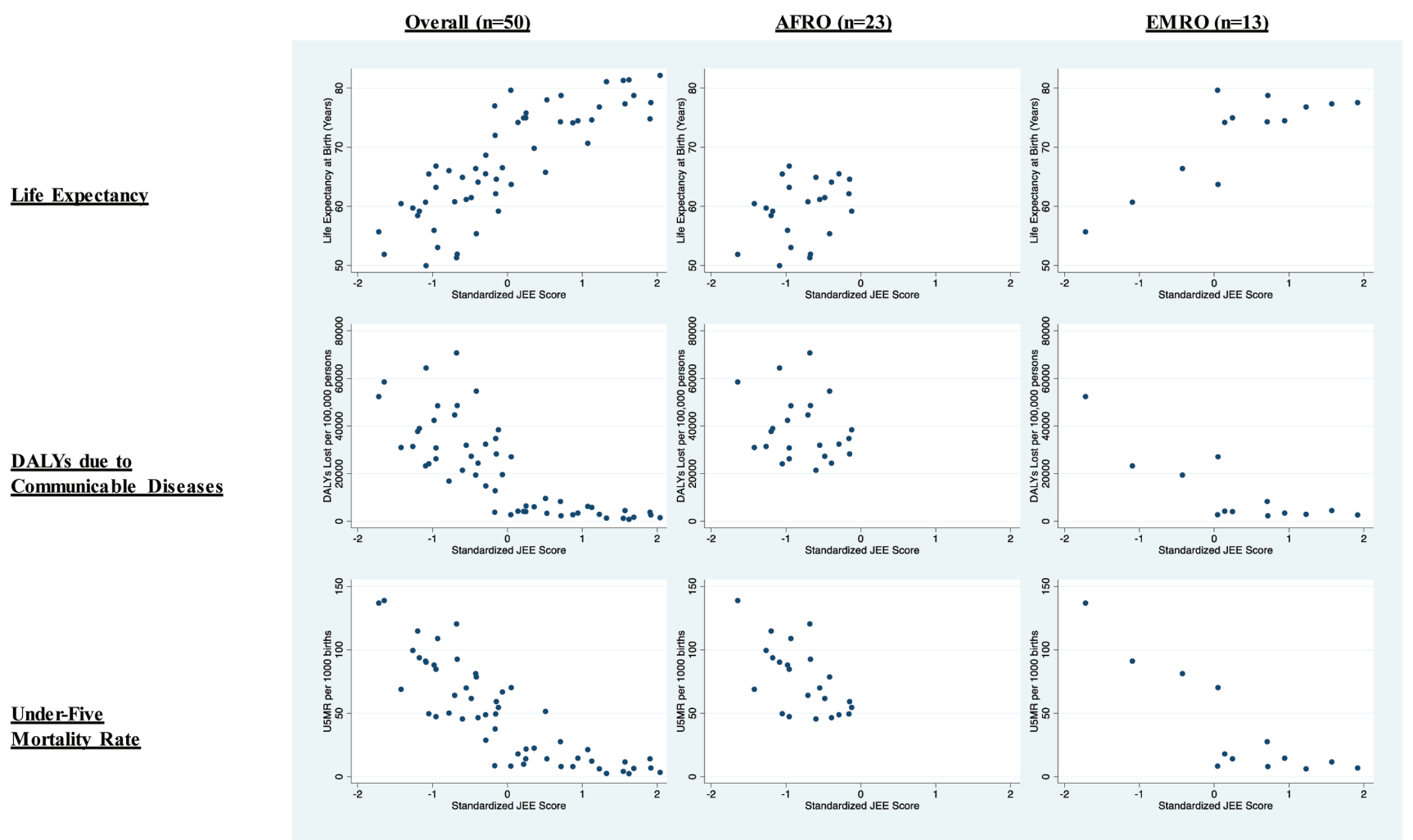

Figure 2. Scatter plots of various demographic characteristics with composite Joint External Evaluation (JEE) performance across various country groupings.

was significantly correlated with under-five mortality $(\rho=-0.80,95 \% \mathrm{CI}=0.97$ to -0.25$)$ and lost DALYs to communicable diseases ( $\rho=-0.65,95 \% \mathrm{CI}=-0.92$ to -0.01 ).

Among enabling factors, statistically significant correlations were observed within the overall sample for GDP per capita ( $\rho=0.81,95 \% \mathrm{CI}=0.68$ to 0.89$)$, public health expenditures as a percentage of GDP ( $\rho=0.36,95 \%=$ CI 0.10 to 0.58 ), and density of skilled health professionals per 10000 persons ( $\rho=0.81$, $95 \%=$ CI 0.71 to 0.89 ) (Table 3 and Figure 3). No enabling factor was significantly correlated with JEE score in AFRO, but GDP per capita was correlated with JEE performance in EMRO ( $\rho=0.85,95 \%$ $\mathrm{CI}=0.61$ to 0.99 ). 


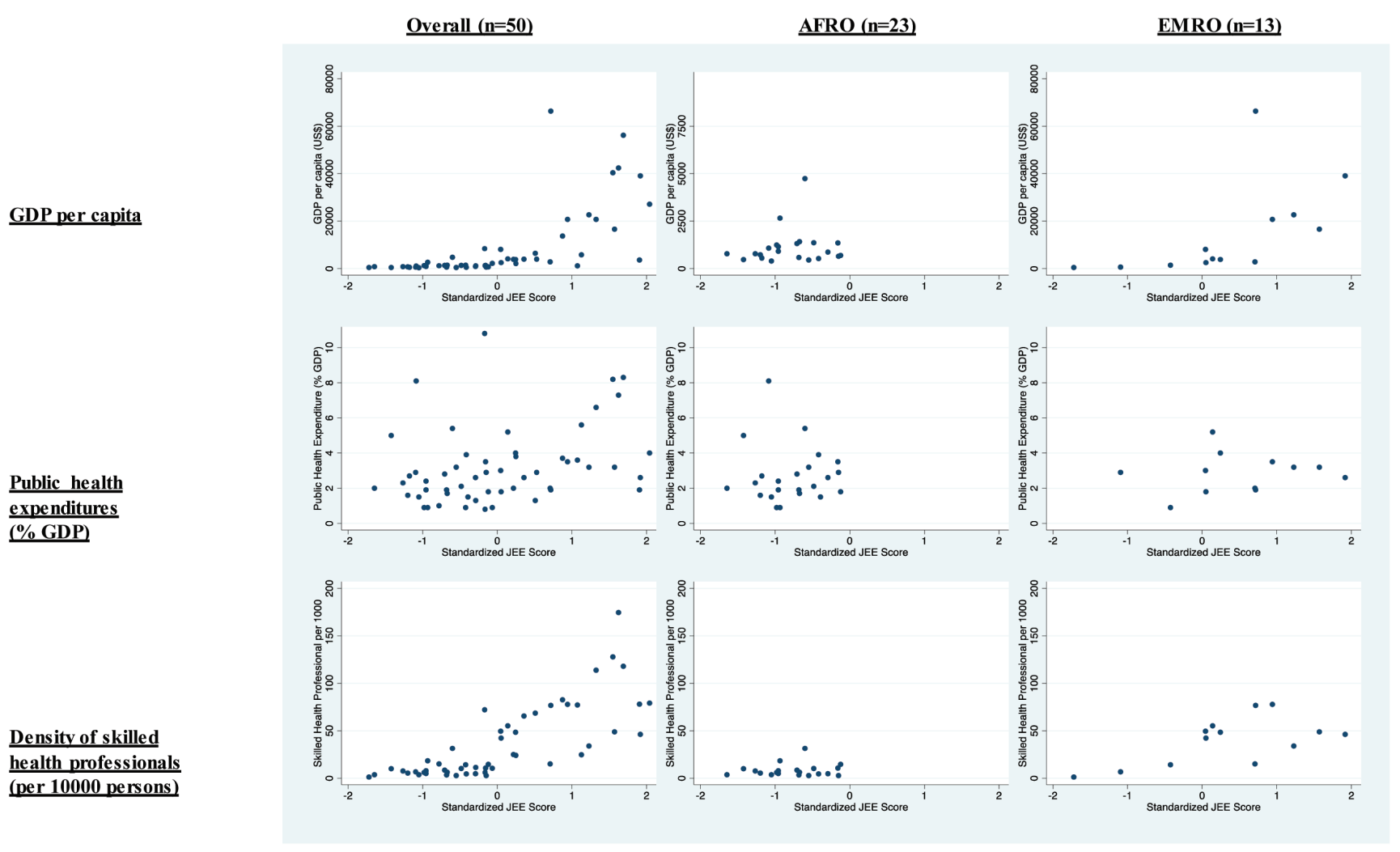

Figure 3. Scatter plots of various enabling factors with composite Joint External Evaluation (JEE) performance across various country groupings.

\section{DISCUSSION}

We examined performance on the Joint External Evaluation, the WHO's voluntary tool for assessing IHR core capacity and preparedness to potential public health threats, among the initial 55 countries that have undergone the assessment. These evaluations reveal large gaps in core public health capacity and preparedness across a range of indicators with a large majority of countries scoring less than 4 (indicating non-sustainable or undeveloped capacities) on a majority of indicators. Antimicrobial stewardship and surveillance for highly resistant pathogens were areas of particular concern though there were other areas with substantial challenges as well. There were large variations in performance by geography, with countries from WHO AFRO and SEARO regions scoring lowest on the JEE and those in PAHO and EURO scoring highest. Taken together, these findings are a critical wake-up call that while JEE assessments are critical, they are only a first step towards making the kinds of financial and technical investments necessary to help countries improve their public health capacities.

Most of the capacities measured by the indicators in the JEE represent essential public health functions, such as disease surveillance, laboratory capacity, and sufficient human resources. These combine to create an infrastructure which protects the health of a country's population from threats such as communicable zoonotic diseases, food poisoning and waterborne illnesses, allowing nations to contain public health emergencies at their source. The poor performance on many of these indicators (scores of less than 4) suggest that countries are not only ill-prepared for cross-border outbreaks but are struggling to provide key public health services that are critical to keeping their populations healthy and safe. Without these core capacities in place, future outbreaks may become large-scale pandemics. Reasons for poor capacity are clearly multifactorial, as many countries exhibit broad-based weaknesses across a range of indicators. One fact is clear from these analyses: further investments are needed that specifically target preparedness and core public health functions represented by the JEE.

Yet, success in building any capacity can only be achieved with leadership and investment of resources, prioritization, and commitment by the specific countries themselves. Few countries completely lack ca- 
pacity but appear to have some existing infrastructure and competence on which to build. Not surprisingly, resource-constrained regions of the world performed less well on the JEE than relatively affluent regions like PAHO, EURO and parts of EMRO and WPRO. Further, poor health outcomes, as measured by DALYs due to communicable disease, are more frequent in AFRO and SEARO, which have high burdens of disease while also exhibiting lower life expectancy and higher mortality rates. The observation that JEE performance seems to correlate well with these baseline health, demographic and economic data points suggests that this tool is accurately measuring its intended core public health-related capabilities.

Indeed, the JEE does appear to correlate well with the gross overview of the strength and performance of the core public health components of health systems as defined by life expectancy, under 5-year old mortality, and disability due to communicable disease. Moreover, performance on the JEE positively correlated with the presence of enabling resources for health (high levels of GDP per capita and skilled health professionals). However, less consistent correlations in region-specific analyses highlight a need to further validate the JEE tool across contexts and suggests value for context-specific prioritization of functions. Overall, our findings suggest the JEE tool accurately measures relevant health security capabilities, though longitudinal studies will be needed to confirm this. A slightly revised update of the tool was recently published by the WHO, but changes are small and thus unlikely to affect our results. So far, no JEE reports using the revised tool have been published.

There are important limitations to our work. First, JEE reports have only been conducted on 28.2\% $(n=55)$ of the WHO Member States; further, each evaluated country has had only one assessment. Also, for some specific activities, such as prevention of antimicrobial drug resistance, interventions have started only relatively recently. Thus, the limitations of a cross-sectional analysis apply here and diminish the generalizability of our findings, since we expect variation in JEE performance over time to be more informational in guiding improvement. Further, there is asymmetric representation from each WHO region, which may have led to an inaccurate estimation of regional trends in JEE performance; however, with our adjustments, we believe this initial analysis will provide helpful guidance to policymakers as an interim summary on JEE results to-date.

Moreover, our analysis is not intended to serve as a definitive validation exercise, nor are we aiming to assess cause and effect between JEE scores and outcomes. Our relatively small data set handicapped our ability to pursue more advanced statistical models that may better identify possible predictors of JEE performance. As more analyses are performed, and particularly as countries go through additional iterations of the assessment over time, we believe pursuing such analyses in the future will be both helpful and appropriate.

In conclusion, core public health functions and preparedness remains broadly weak across a range of indicators among a moderately large sample of WHO member countries, though substantial regional variations exist. Capabilities in AMR and emergency response are among the lowest scoring, and our results suggest that regions with greater health resources exhibit improved performance on the JEE. We do find, however, that the JEE tool itself and the process it is applied by are important and seemingly effective in measuring the strength of the core public health components of health systems. As global generic indicators for these functions remain sparse and underutilized, the JEE process provides an additional opportunity to measure the impact of investing in essential public health functions.

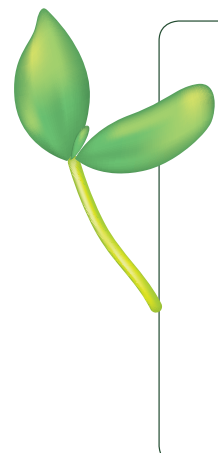

\section{Acknowledgements: None.}

Ethical issues: This study was exempt from review by our respective institutions' research review boards.

Funding: No sources of funding were utilized in the performance of this study.

Authorship contributions: MS, VG, JDK and RK designed the study and statistical analysis as well as contributed to the drafting of the manuscript, assisted by AKJ and VBK. JS and JO assisted with data collection and the statistical design of the study as well as provided some comments to the draft. VG wrote the first draft and led the overall analysis.

Competing interests: The authors have completed the Unified Competing Interest form at www.icmje.org/ coi_disclosure.pdf (available on request from the corresponding author) and declare no conflict of interest. 
1 Katz RL, Fernandez JA, McNabb SJ. Disease surveillance, capacity building and implementation of the International Health Regulations (IHR[2005]). BMC Public Health. 2010;10 Suppl 1:S1. Medline:21143819 doi:10.1186/1471-245810-S1-S1

2 Fidler DP, Gostin LO. The new International Health Regulations: an historic development for international law and public health. J Law Med Ethics. 2006;34:85-94. Medline:16489987 doi:10.1111/j.1748-720X.2006.00011.x

3 World Health Organization. International Health Regulations (2005). Geneva: WHO;2016.

4 Baker MG, Fidler DP. Global public health surveillance under new International Health Regulations. Emerg Infect Dis. 2006;12:1058-65. Medline:16836821 doi:10.3201/eid1207.051497

5 Kimball AM, Heymann D. Ebola, International Health Regulations, and global safety. Lancet. 2014;384:2023. Medline:25483162 doi:10.1016/S0140-6736(14)62330-8

6 Carafano JJ, Florance C, Kaniewski D. The Ebola outbreak of 2013-2014: An assessment of U.S. Actions. Special Report of The Heritage Foundation. 2015;166:13-21.

7 Gates B. The next epidemic — Lessons from Ebola. N Engl J Med. 2015;372:1381-4. Medline:25853741 doi:10.1056/ NEJMp1502918

8 Heymann DL, Chen L, Takemi K, Fidler DP, Tappero JW, Thomas MJ, et al. Global health security: The wider lessons from the west African Ebola virus disease epidemic. Lancet. 2015;385:1884-901. Medline:25987157 doi:10.1016/ S0140-6736(15)60858-3

9 Katz R, Seifman R. Opportunities to finance pandemic preparedness. Lancet Glob Health. 2016;4:e782-3. Medline:27692862 doi:10.1016/S2214-109X(16)30202-9

10 Graves CM, Haakenstad A, Dieleman JL. Tracking development assistance for health to fragile states: 2005-2011. Global Health. 2015;11:12. Medline:25886046 doi:10.1186/s12992-015-0097-9

11 Dieleman JL, Schneider MT, Haakenstad A, Singh L, Sadat N, Birger M, et al. Development assistance for health: past trends, associations, and the future of international financial flows for health. Lancet. 2016;387:2536-44. Medline:27086170 doi:10.1016/S0140-6736(16)30168-4

12 Maciosek MV, Coffield AB, Flottemesch TJ, Edwards NM, Solberg LI. Greater use of preventive services in U.S. health care could save lives at little or no cost. Health Aff (Millwood). 2010;29:1656-60. Medline:20820022 doi:10.1377/ hlthaff.2008.0701

13 World Health Organization. Implementation of the International Health Regulations (2005): report of the Review Committee on Second Extensions for Establishing National Public Health Capacities and on IHR Implementation: report by the Director General. Geneva: WHO; 2015.

14 Gostin LO, Katz R. The International Health Regulations: The governing framework for global health security. Milbank Q. 2016;94:264-313. Medline:27166578 doi:10.1111/1468-0009.12186

15 World Health Organization. Development, monitoring, and evaluation of functional core capacity for implementing the International Health Regulations (2005). Geneva: WHO; 2015.

16 World Health Organization. Technical consultation on monitoring and evaluation of functional core capacity for implementing the International Health Regulations (2005). Geneva: WHO; 2015.

17 World Health Organization. Strategic Partnership Portal | Building Stronger Health Systems for Sustainable Health Security. 2017. Available: https://extranet.who.int/spp/ihrmef. Accessed: 5 May 2018.

18 World Health Organization. Joint External Evaluation Tool. Available: http://apps.who.int/iris/bitstre am/10665/204368/1/9789241510172_eng.pdf. Accessed: 5 May 2018.

19 World Health Organization. Sixty-eighth World Health Assembly: Report of the Review Committee on Second Extensions for Establishing National Public Health Capacities and on IHR Implementation. Geneva: WHO; 2015.

20 World Health Organization. Sixty-eighth World Health Assembly: Ebola Interim Assessment Panel Report by the Secretariat. Geneva: WHO; 2015.

21 World Health Organization. Sixty-ninth annual World Health Assembly: Implementation of the International Health Regulations (2005) Ebola virus disease. Geneva: WHO; 2016.

22 Kandel N, Sreedharan R, Chungong S, Sliter K, Nikkari S, Ijaz K, et al. Joint external evaluation process: bringing multiple sectors together for global health security. Lancet Glob Health. 2017;5:e857-8. Medline:28807175 doi:10.1016/ S2214-109X(17)30264-4

23 World Health Organization. Country JEE Mission Reports. Available: http://www.who.int/ihr/procedures/mission-reports/en/. Accessed: 5 May 2018.

24 Bell E, Tappero JW, Iljaz K, Bartee M, Fernandez J, Burris H, et al. Joint External Evaluation—Development and scaleup of global multisectoral health capacity evaluation process. Emerg Infect Dis. 2017;23:S33-7. Medline:29155678 doi:10.3201/eid2313.170949

25 Balabanova D, Mills A, Conteh L, Akkazieva B, Banteyerga H, Dash U, et al. Good health at low cost 25 years on: Lessons for the future of health systems strengthening. Lancet. 2013;381:2118-33. Medline:23574803 doi:10.1016/S01406736(12)62000-5

26 OECD/EU. Health at a Glance: Europe 2016: State of Health in the EU Cycle. Paris: OECD; 2016.

27 Kolenikov S, Angeles G. Socioeconomic status measurement with discrete proxy variables: is principal components analysis a reliable answer? Rev Income Wealth. 2009;55:128-65. doi:10.1111/j.1475-4991.2008.00309.x

28 DiCiccio TJ, Efron B. Bootstrap confidence intervals. Stat Sci. 1995;11:189-212. 